\title{
High-sensitive C-reactive protein and stroke outcomes in patients with and without atrial fibrillation.
}

\author{
Tieyu Tang ${ }^{1 \#}$, Zuowei Duan ${ }^{1 \#, ~ C h a n g b i a o ~ F u ¹, ~ Z h e n s h e n g ~ L i u ², ~ K a i z h e n g ~ G o n g ~}{ }^{3}$, Xinjiang Zhang ${ }^{\text {* }}$ \\ ${ }^{1}$ Department of Neurology, the First People's Hospital of Yangzhou, Yangzhou, PR China \\ ${ }^{2}$ Department of Radiology, the First People's Hospital of Yangzhou, Yangzhou, PR China \\ ${ }^{3}$ Department of Cardiology, the First People's Hospital of Yangzhou, Yangzhou, PR China \\ \#These authors contributed equally to this work
}

\begin{abstract}
Aims: The purpose of this study was to examine and compare the association of baseline high-sensitive C-reactive protein (hsCRP) levels with Early Neurological Deterioration (END) in acute ischemic stroke with and without Atrial Fibrillation (AF).

Methods: Consecutive ischemic stroke patients admitted to our center within $24 \mathrm{~h}$ of symptom onset were prospectively recruited. Plasma hsCRP levels were measured after admission and categorized into three groups (low risk, $<1.0 \mathrm{mg} / \mathrm{L}$; average risk, $1-3 \mathrm{mg} / \mathrm{L}$; and high risk, $>3 \mathrm{mg} / \mathrm{L}$ ). END was defined as a National Institutes of Health Stroke Scale (NIHSS) score increased $\geq 2$ during the first 72 h compared with the initial NIHSS score.

Results: A total of 811 patients with ischemic stroke including 348 female with average age of $66.44 \pm$ $12.54 \mathrm{y}$ were enrolled in the analysis. Of them, AF was found in 226 (27.9\%) patients. During the first 72 $h$ after admission, 81 (35.8\%) patients with AF and 181 (30.9\%) patients without AF developed END, respectively. After adjusting the confounding factors whose $P$ value $<0.1$ in univariate analysis, multiple logistic regression analysis demonstrated that high risk level of hsCRP $(\mathrm{OR}=2.39,95 \%$ CI: 1.06-5.40) was the independent predictors of END in AF-related stroke. However, any level of hsCRP was found to be independently related to END in patients without AF.

Conclusion: Our study demonstrated that high level of hsCRP independently correlated with END in AF-related ischemic stroke.
\end{abstract}

Keywords: Stroke, C-reactive protein, Early neurological deterioration, Inflammation.

\section{Introduction}

Atrial Fibrillation (AF) is associated with an increased risk of ischemic stroke and AF-related stroke is related to more disabling and unfavorable outcomes compared with those caused by other stroke pathophysiology [1,2]. Previous studies had suggested increased risk of thrombogenesis in participates with AF [3]. And this thrombogenic tendency was considered to be related to systemic inflammatory conditions [4]. As a blood marker of inflammation, high-sensitive C-reactive protein (hsCRP), an acutephase protein, had been reported to be increased in $\mathrm{AF}$, and predict new-onset $\mathrm{AF}$ and $\mathrm{AF}$ recurrence after cardioversion or catheter ablation [5-7]. Furthermore, hsCRP had been reported to predict mid- and long-term prognosis in patients with ischemic stroke [8-10]. Early Neurological Deterioration (END), a known complication of stroke in general, commonly occurs in hours or even days after symptom onset and often leads to worse clinical outcome, may also be related to hsCRP $[11,12]$.
However, this finding still lacks confirmation by other groups. And whether hsCRP predicts END differently in AF-related versus non-AF-related stroke is largely unknown. Currently, precise pathophysiology of END is yet incompletely elucidated. Thus, identification of various predictors for END in a certain population might help in mitigating its occurrence and influence on functional outcome. Therefore, we sought to examine and compare the association between baseline hsCRP level and END in acute ischemic stroke with and without AF.

\section{Materials and Methods}

\section{Subjects}

This study reviewed consecutive patients with a diagnosis of ischemic stroke who were admitted to our hospital within $24 \mathrm{~h}$ of symptom onset between January 2014 and January 2016. All patients included in this study were more than 18 years old. For those patients with stroke on awakening, stroke onset was 
designated as the halfway point between the time when the patient was last symptom-free and when the neurologic deficits became apparent. Ischemic stroke was defined as the presence of focal neurologic deficits explained by the relevant lesions detected on brain Magnetic Resonance Imaging (MRI) performed within $24 \mathrm{~h}$ after admission. Reasons for exclusion from the study included intracranial hemorrhage, tumors and infections and intravenous or intra-arterial thrombolysis, incomplete medical records, and early discharge from the hospital (within 3 or fewer days). The study was approved by Ethics Committees of the First People's Hospital of Yangzhou and all subjects provided written informed consent before entering the study.

\section{Clinical course assessment}

Detailed data were prospectively recorded including demographics, medical history and associated cardiovascular risk factors, stroke mechanism and laboratory findings. Hypertension was defined as systolic blood pressure $\geq 140 \mathrm{~mm}$ $\mathrm{Hg}$ or diastolic blood pressure $\geq 90 \mathrm{~mm} \mathrm{Hg}$ in the chronic stage, or as a previous history of treatment with antihypertensive drugs. Diabetes mellitus was determined by a fasting blood glucose $\geq 126 \mathrm{mg} / \mathrm{dl}$, positive $\geq 75 \mathrm{~g}$ oral glucose tolerance test result, or use of insulin or oral hypoglycemic agents. Smoking was defined as current or former cigarette smoking, and alcohol intake was defined as habitual consumption of alcohol beverages before onset of stroke. Data on history of stroke was also collected.

Information of AF was evaluated by the study cardiologist, and AF was diagnosed based on electrocardiographic findings on admission or during hospitalization, or a self-reported history of AF [13,14]. When AF was strongly suspected from the clinical presentation and brain imaging findings, repeated electrocardiogram or $24 \mathrm{~h}$ Holter electrocardiographic monitoring was conducted during hospital stay.

Levels of hsCRP, total cholesterol, triglycerides, Low-Density Lipoprotein (LDL), and High Density Lipoprotein (HDL), Fasting Blood-Glucose (FBG), Blood Urea Nitrogen (BUN) and Creatinine $(\mathrm{Cr})$ were also analysed from fasting blood samples obtained within $24 \mathrm{~h}$ of admission. hsCRP level was analysed and divided into three categories based on the relative risk category recommended by the Centers for Disease Control and American Heart Association (low risk, $<1.0 \mathrm{mg} / \mathrm{L}$; average risk, 1-3 $\mathrm{mg} / \mathrm{L}$; and high risk, $>3 \mathrm{mg} / \mathrm{L})$, originally recommended for the risk assessment of cardiovascular disease [15].

\section{Imaging analysis}

A comprehensive MRI protocol including T1WI, T2WI, DWI, fluid-attenuated inversion recovery (FLAIR), and 3D time-offlight Magnetic Resonance Angiography (MRA) was carried out by either $1.5 \mathrm{~T}$ (Signa; GE; USA) or a 3.0 T (Magnetom Avanto; Siemens, Germany) system largely depending on which one was available to achieve quick evaluation during the first $24 \mathrm{~h}$ after admission. Then Computer Tomography
Angiography (CTA) was also performed to evaluate the status of craniocervical artery. Catheter-based Digital Subtraction Angiography (DSA) was performed only if these noninvasive angiographies were unable to provide sufficient information for conclusive diagnosis. Based on these imaging data, two experienced neuroradiologists, who were blinded to the patients' clinical details, independently evaluated the size of the infarction and the status of craniocervical artery. The size of the lesion was represented by the largest diameter of the lesion on DWI. The intracranial stenosis and extracranial stenosis were evaluated according to WASID criteria and NASCET criteria, respectively $[16,17]$. Consensus was reached over inter-observer discrepancies through consultation.

\section{Treatment protocol and neurological deficits assessment}

Once admitted in the stroke unit, guideline-based treatments were immediately started. Two basic of antithrombotic therapies including antiplatelet and anticoagulation were selected largely based on the stoke mechanisms and stroke unit's therapeutic and diagnostic protocol $[18,19]$. Oral stain was mandatory administrated in case of no contradictions. In addition, other treatments such as blood pressure, glucose management were also carried out as appropriate.

Neurological deficits assessment was performed using the National Institutes of Health Stroke Scale (NIHSS) at admission and continued at the following $72 \mathrm{~h}$ by a certified neurologist blind to clinical and imaging information. END was defined as the subsequent NIHSS score increasing $\geq 2$ points compared with initial one [20,21].

\section{Statistical analysis}

All statistical analyses were performed using SPSS version 16.0. A $\mathrm{P}$ value $<0.05$ was considered significant. Continuous data were described by their mean \pm SD or median (Interquartile Range (IQR)) and categorical data as proportions (percentage). For univariate analysis, student t-test, variance analysis, Mann-Whitney test, $\chi^{2}$-test or Fisher's exact test was performed as appropriate. Multiple logistic regression model was performed using variables with $\mathrm{P}$ value $\leq 0.1$ in univariate analysis to determine the independent predictors of END. The results were calculated as adjusted Odds Ratios (ORs) with 95\% Confidence Intervals (CIs).

\section{Results}

A total of 811 patients with ischemic stroke including 348 female with average age of $66.44 \pm 12.54 \mathrm{y}$ were enrolled in the analysis. The median NIHSS score at admission was 7 (interquartile range, 3 to 11). Of them, AF was found in 226 $(27.9 \%)$ patients. Other risk factors included hypertension 559 $(68.9 \%)$, diabetes mellitus in $202(24.9 \%)$, smoking in 230 $(28.4 \%)$, and drinking in $137(16.9 \%)$. Patients with responsible artery occlusion were found in 120 (14.8) patients 
and responsible artery stenosis in 375 (46.2). Previous TIA or stroke was founded in $109(13.4 \%)$ patients (Table 1$)$.

Table 1. Basic clinical data of patients.

\begin{tabular}{|c|c|c|c|}
\hline Characteristics & All patients $(\mathrm{N}=\mathbf{8 1 1})$ & Patients with AF ( $N=226)$ & Patients without AF $(\mathrm{N}=585)$ \\
\hline \multicolumn{4}{|l|}{ General clinical characteristics } \\
\hline Age, years, mean (SD) & $66.44 \pm 12.54$ & $68.79 \pm 10.96$ & $65.33 \pm 13.09$ \\
\hline Female, N (\%) & 348 (42.9) & $96(42.5)$ & $252(43.13)$ \\
\hline Hypertension, N (\%) & $559(68.9)$ & $162(71.7)$ & $397(67.9)$ \\
\hline Diabetes mellitus, $\mathrm{N}(\%)$ & $202(24.9)$ & $52(23.0)$ & $150(25.6)$ \\
\hline Previous TIA or stroke, N (\%) & $109(13.4)$ & $46(20.4)$ & $63(10.8)$ \\
\hline Smoking, N (\%) & $230(28.4)$ & $60(26.5)$ & $170(29.1)$ \\
\hline Drinking, N (\%) & $137(16.9)$ & $35(15.5)$ & $102(17.4)$ \\
\hline Initial NIHSS, median (IQR) & $7(3-11)$ & $8(5-12)$ & $6(3-11)$ \\
\hline \multicolumn{4}{|l|}{ Hematological parameters } \\
\hline WBC $\left(10^{9}, \bar{x} \pm s\right)$ & $6.87 \pm 2.36$ & $6.92 \pm 2.03$ & $6.84 \pm 2.48$ \\
\hline $\operatorname{RBC}\left(10^{12}, \bar{x} \pm s\right)$ & $4.52 \pm 0.64$ & $4.55 \pm 0.72$ & $4.51 \pm 0.59$ \\
\hline $\operatorname{BUN}(\mathrm{mmol} / \mathrm{l}, \overline{\mathrm{x}} \pm \mathrm{s})$ & $5.44 \pm 2.76$ & $5.54 \pm 3.84$ & $5.39 \pm 2.00$ \\
\hline $\mathrm{Cr}(\mathrm{mmol} / \mathrm{l}, \overline{\mathrm{x}} \pm \mathrm{s})$ & $76.64 \pm 34.97$ & $77.55 \pm 36.62$ & $76.21 \pm 34.18$ \\
\hline FBG $(\mathrm{mmol} / \mathrm{l}, \bar{x} \pm \mathrm{s})$ & $6.48 \pm 2.33$ & $6.44 \pm 2.49$ & $6.49 \pm 2.27$ \\
\hline Total cholesterol (mmol/l, $\bar{x} \pm \mathrm{s})$ & $4.68 \pm 1.17$ & $4.77 \pm 1.28$ & $4.63 \pm 1.11$ \\
\hline Triglycerides $(\mathrm{mmol} / \mathrm{l}, \overline{\mathrm{x}} \pm \mathrm{s})$ & $1.61 \pm 1.08$ & $1.62 \pm 1.24$ & $1.60 \pm 1.00$ \\
\hline LDL-cholesterol $(\mathrm{mmol} / \mathrm{I}, \overline{\mathrm{x}} \pm \mathrm{s})$ & $2.67 \pm 0.91$ & $2.86 \pm 0.91$ & $2.60 \pm 0.89$ \\
\hline HDL-cholesterol (mmol/l, $\bar{x} \pm s)$ & $1.19 \pm 0.31$ & $1.20 \pm 0.32$ & $1.19 \pm 0.31$ \\
\hline \multicolumn{4}{|l|}{ hsCRP } \\
\hline Low risk, $\mathrm{N}(\%)$ & $305(37.6)$ & $64(28.3)$ & $241(41.2)$ \\
\hline Average risk, $\mathrm{N}(\%)$ & $239(29.5)$ & $70(31.5)$ & $169(28.9)$ \\
\hline High risk, $\mathrm{N}(\%)$ & $267(32.9)$ & $92(40.7)$ & $175(29.9)$ \\
\hline \multicolumn{4}{|l|}{ Imaging indexes } \\
\hline Lesion $\geq 20$ mm, N (\%) & $272(33.5)$ & $80(35.4)$ & $192(32.8)$ \\
\hline Multiple lesion, N (\%) & $173(21.3)$ & $57(25.2)$ & $116(19.8)$ \\
\hline Responsible artery occlusion, N (\%) & $120(14.8)$ & $49(21.7)$ & $71(12.1)$ \\
\hline Responsible artery stenosis, N (\%) & $375(46.2)$ & $105(46.5)$ & $270(46.2)$ \\
\hline \multicolumn{4}{|l|}{ Current medications } \\
\hline Antiplatelet age, $\mathrm{N}(\%)$ & $681(84.0)$ & $182(80.5)$ & $499(85.3)$ \\
\hline Anticoagulants, $\mathrm{N}(\%)$ & $42(5.2)$ & $29(12.8)$ & $13(2.2)$ \\
\hline END, N (\%) & $262(32.3)$ & $81(35.8)$ & $181(30.9)$ \\
\hline
\end{tabular}

AF: Atrial Fibrillation; hsCRP: High-Sensitivity C-Reactive Protein; WBC: White Blood Cell; RBC: Red Blood Cell; LDL: Low-Density Lipoprotein; HDL: High-Density Lipoprotein; FBG: Fasting Blood-Glucose; BUN: Blood Urea Nitrogen; Cr: Creatinine; END: Early Neurological Deterioration.

Low risk level of hsCRP was found in 305 (37.6\%) patients.

Average risk and high risk level of hsCRP were found in 239
$(29.5 \%)$ and $267(32.9 \%)$ patients, respectively. Table 2 shows the clinical characteristics of patients according to the hsCRP 
risk level. The age, initial NIHSS score, FBG and END were significantly different between patients with different level of hsCRP $(\mathrm{P}<0.05)$.

Table 2. Comparisons of basic characteristics of patients according to hsCRP level.

\begin{tabular}{|c|c|c|c|}
\hline Characteristics & hsCRP<1 mg/L N=305 & hsCRP 1-3 mg/L N=239 & hsCRP>3 mg/L N=267 \\
\hline \multicolumn{4}{|l|}{ General clinical characteristics } \\
\hline Age, years, mean (SD) & $64.88 \pm 12.58$ & $66.67 \pm 11.59$ & $68.03 \pm 2.46^{*}$ \\
\hline Female, N (\%) & $125(41.0)$ & $101(42.3)$ & $122(45.7)$ \\
\hline Hypertension, N (\%) & $204(66.9)$ & $162(67.8)$ & $193(72.3)$ \\
\hline Diabetes mellitus, $\mathrm{N}(\%)$ & $64(21.0)$ & $62(25.9)$ & $76(28.5)$ \\
\hline Previous TIA or stroke, $\mathrm{N}(\%)$ & $35(11.5)$ & $27(11.3)$ & $47(17.6)^{\star *}$ \\
\hline Smoking, N (\%) & $77(25.2)$ & $73(30.5)$ & $80(30.0)$ \\
\hline Drinking, N (\%) & $51(16.7)$ & $45(18.8)$ & $41(15.4)$ \\
\hline Initial NIHSS, median (IQR) & $5(2-9)$ & $7(4-12)$ & $9(4-12)^{*}$ \\
\hline \multicolumn{4}{|l|}{ Hematological parameters } \\
\hline WBC $\left(10^{9}, \bar{x} \pm s\right)$ & $6.77 \pm 2.41$ & $6.76 \pm 2.26$ & $7.08 \pm 2.38^{* *}$ \\
\hline $\operatorname{RBC}\left(10^{12}, \bar{x} \pm s\right)$ & $4.52 \pm 0.66$ & $4.52 \pm 0.65$ & $4.53 \pm 0.60$ \\
\hline $\mathrm{BUN}(\mathrm{mmol} / \mathrm{l}, \overline{\mathrm{x}} \pm \mathrm{s})$ & $5.52 \pm 3.75$ & $5.16 \pm 1.65$ & $5.60 \pm 2.13$ \\
\hline $\mathrm{Cr}(\mathrm{mmol} / \mathrm{l}, \overline{\mathrm{x}} \pm \mathrm{s})$ & $77.04 \pm 37.20$ & $72.69 \pm 23.94$ & $79.72 \pm 40.06^{* *}$ \\
\hline $\mathrm{FBG}(\mathrm{mmol} / \mathrm{l}, \overline{\mathrm{x}} \pm \mathrm{s})$ & $6.33 \pm 2.16$ & $6.33 \pm 2.26$ & $6.78 \pm 2.54^{*}$ \\
\hline Total cholesterol (mmol/l, $\bar{x} \pm \mathrm{s})$ & $4.70 \pm 1.16$ & $4.63 \pm 1.28$ & $4.69 \pm 1.08$ \\
\hline Triglycerides $(\mathrm{mmol} / \mathrm{l}, \overline{\mathrm{x}} \pm \mathrm{s})$ & $1.63 \pm 1.21$ & $1.60 \pm 0.87$ & $1.60 \pm 1.11$ \\
\hline LDL-cholesterol (mmol/l, $\bar{x} \pm \mathrm{s})$ & $2.64 \pm 0.88$ & $2.66 \pm 0.97$ & $2.71 \pm 0.88$ \\
\hline HDL-cholesterol (mmol/l, $\bar{x} \pm s)$ & $1.21 \pm 0.30$ & $1.17 \pm 0.27$ & $1.19 \pm 0.35$ \\
\hline \multicolumn{4}{|l|}{ Imaging indexes } \\
\hline Lesion $\geq 20 \mathrm{~mm}, \mathrm{~N}(\%)$ & $105(34.4)$ & $70(29.3)$ & $97(36.3)$ \\
\hline Multiple lesion, N (\%) & $65(21.3)$ & $48(20.1)$ & $60(22.5)$ \\
\hline Responsible artery occlusion, N (\%) & $40(13.1)$ & $33(13.8)$ & $47(17.6)$ \\
\hline Responsible artery stenosis, N (\%) & $149(48.9)$ & $104(43.5)$ & $122(45.7)$ \\
\hline \multicolumn{4}{|l|}{ Current medications } \\
\hline Antiplatelet age, N (\%) & $249(81.6)$ & $204(85.4)$ & $228(85.4)$ \\
\hline Anticoagulants, N (\%) & $21(6.9)$ & $11(4.6)$ & $10(3.7)$ \\
\hline END, N (\%) & $75(24.6)$ & $80(33.5)$ & $107(40.1)^{*}$ \\
\hline
\end{tabular}

AF: Atrial Fibrillation; hsCRP: High-Sensitivity C-Reactive Protein; WBC: White Blood Cell; RBC: Red Blood Cell; LDL: Low-Density Lipoprotein; HDL: High-Density Lipoprotein; FBG: Fasting Blood-Glucose; BUN: Blood Urea Nitrogen; Cr: Creatinine; END: Early Neurological Deterioration.

${ }^{*} \mathrm{P}<0.05 ;{ }^{* *} \mathrm{P}<0.01$

Of the 811 subjects included in the study, 226 patients were diagnosed with AF. During the first $72 \mathrm{~h}$ after admission, 81 (35.8\%) patients with AF and 181 (30.9\%) patients without AF developed END, respectively. After adjusting the confounding factors (including age, sex, previous TIA or stroke, baseline NIHSS, LDL, FBG and responsible artery occlusion ) whose $\mathrm{P}$ value $\leq 0.1$ in univariate analysis, multiple logistic regression analysis demonstrated that, compared with low risk level of hsCRP, high risk level of hsCRP $(\mathrm{OR}=2.39,95 \% \mathrm{CI}$ : 1.06-5.40) was the independent predictors of END in AFrelated ischemic stroke. For those without AF, however, any level of hsCRP was found to be independently related to END 
after adjusting the age, sex, diabetes mellitus, smoking, baseline NIHSS, LDL, FBG and responsible artery occlusion (Table 3).

Table 3. Multivariable analysis of the associations between hSCRP levels and the development of END.

\begin{tabular}{|c|c|c|c|c|}
\hline & Unadjusted OR $(95 \% \mathrm{CI})$ & Adjusted $^{\dagger}$ OR $(95 \% \mathrm{Cl})$ & Unadjusted OR (95\% Cl) & Adjusted OR ${ }^{\ddagger}(95 \% \mathrm{Cl})$ \\
\hline \multicolumn{5}{|l|}{ hsCRP } \\
\hline Low risk & Ref & Ref & & \\
\hline Average risk & $2.32(1.06-5.05)$ & $1.97(0.86-4.54)$ & $1.36(0.88-2.09)$ & $1.28(0.81-2.02)$ \\
\hline High risk & $3.30(1.58-6.87)$ & $2.39(1.06-5.40)$ & $1.71(1.12-2.60)$ & $1.37(0.88-2.15)$ \\
\hline
\end{tabular}

${ }^{\dagger}$ Adjustment by age, sex, previous TIA or stroke, baseline NIHSS, LDL, FBG and responsible artery occlusion. ${ }^{\ddagger}$ Adjustment by age, sex, diabetes mellitus, smoking, baseline NIHSS, LDL, FBG and responsible artery occlusion.

\section{Discussion}

The present study analysed a large series of consecutive stroke patients to investigate the role of hsCRP in the prediction of END. We showed that high risk level of hsCRP measured at admission was an independent predictor of END in patients with AF, but not in patients without AF. To prove these associations, we adopted a relatively stringent definition of END as a $\geq 2$-point increase in NIHSS score within $72 \mathrm{~h}$ after admission, which was more likely to be related to hemodynamic and thrombotic causes. Whereas the deterioration in the later stage of ischemic stroke was more association with systemic reasons such as aspiration, infection and electrolyte disturbances. Due to the diverse diagnostic criteria for END and the time interval to evaluation among studies, the rate of END in hospitalized ischemic stroke patients ranged from $12 \%$ to $43 \%$ [20-23]. In the present study, END was present in $32.3 \%$ of the subjects analysed, which was consistent with previous studies.

Inflammation is increasingly considered as playing a central role in the process of atherosclerosis and cardiovascular disease. As one of the most investigated inflammatory makers in cardiovascular researches, hsCRP had been shown to be a useful prognostic indicator for such vascular diseases as myocardial infarction, coronary artery disease, and ischemic stroke [5]. An influence of elevated levels of the CRP on cardiovascular or stroke functional outcome had also been reported [6-10]. In the Framingham Study, elevated plasma CRP levels were shown to be significantly associated with future ischemic stroke and transient ischemic attack independent of conventional risk factors [24]. In addition, a study from the Third National Health and Nutrition Examination Survey investigated the relationship between hsCRP and stroke risk in 880 participants. Baseline hsCRP levels were shown to be predictive of mortality and vascular death in AF but not stroke [25]. However, in another study consisted of biracial US population, baseline hsCRP was shown to be a marker of increased risk of stroke but not in participants with AF despite the fact of having higher levels of
hs-CRP [7]. In a word, it was controversy whether the predictive effects of hsCRP on clinical outcome were the same in patients with AF and those with non-AF. The findings of this study support the different predictive effects of hsCRP on the END within $72 \mathrm{~h}$ in AF versus non-AF. To our best knowledge, few studies had examined and compared the relationship between baseline hsCRP level and END in acute ischemic stroke with and without AF.

Currently, the precise mechanisms underlying the association between plasma hsCRP and clinical outcomes after ischemic stroke had not yet been sufficiently studied. As we all know, once an ischemic event occurs, the expression of inflammatory molecules and activation of quiescent inflammatory cells leads to inflammatory state for several months after initial insult [26]. Furthermore, AF itself may lead to an inflammatory state. And previous studies had already shown that patients with AFrelated stroke compared with other subtypes have significantly higher median levels of multiple inflammatory markers like tumor necrosis factor-alpha, IL-6, and IL-1b [27]. Therefore, the predictive effect of hsCRP on END might be markedly magnified in patients with AF. As expected, we found that high level of hsCRP was associated with END in AF-related stroke. Although the precise mechanisms underlying this association were unclear, there are two possible explanations. The first involves the fluid accumulation and edema, which might lead to exacerbation of brain infarction $[5,12]$. Alternatively, patients with AF were reported to be with increased risk of thrombogenesis. This thrombogenic tendency was considered to be related to abnormal changes in inflammatory biomarkers like hsCRP [5,11]. Therefore, hsCRP might further increase the risk of thrombogenesis in patients with AF, which could lead to a infarct expansion or a new thromboembolic events. However, further researches are needed to confirm our result.

We acknowledge certain limitations of our study. First, the present study was limited by its observational study in a single hospital, which might have generated selection bias compared with a population-wide setting. Second, venous blood was collected after an overnight fast when patients had already 
taken the first dosage of antiplatelet drug. However, we supposed that the administration of first dosage of antiplatelet drug may have little impact on hsCRP according current several published studies $[28,29]$. Third, AF in our study was ascertained by electrocardiographic findings and a selfreported history. Although the self-report of a previous physician diagnosis of $\mathrm{AF}$ could be subject to recall and ascertainment bias. Previous study had shown that self-report of a previous physician diagnosis of AF has similar stroke predictive value as electrocardiogram [7,13]. Forth, we had not conducted the second imaging examination after END, so the specific mechanisms of END were not evidently demonstrated. Further researches were needed to elaborate the underlying mechanisms of END.

In conclusion, we found that high level of hsCRP independently correlated with END in AF-related ischemic stroke, which to some degree demonstrated that hsCRP might be a target for intervention during the acute phase of ischemic stroke, especially of the AF-related ischemic stroke. Development of specific human hsCRP inhibitors might further help elaborate the precise mechanisms underlying this association.

\section{Acknowledgement}

All the authors listed have approved the submitted manuscript and we declare that we have no conflict of interest.

\section{References}

1. Hong HJ, Kim YD, Cha MJ. Early neurological outcomes according to CHADS2 score in stroke patients with nonvalvular atrial fibrillation. Eur J Neurol 2012; 19: 284-290.

2. Ball J, Carrington MJ, McMurray JJ, Stewart S. Atrial fibrillation: profile and burden of an evolving epidemic in the 21 st century. Int J Cardiol 2013; 167: 1807-1824.

3. Watson T, Shantsila E, Lip GY. Mechanisms of thrombogenesis in atrial fibrillation: Virchows triad revisited. Lancet 2009; 373: 155-166.

4. Freestone B, Lip GY. The endothelium and atrial fibrillation. The prothrombotic state revisited. Hamostaseologie 2008; 28: 207-212.

5. Paquissi FC. The predictive role of inflammatory biomarkers in atrial fibrillation as seen through neutrophillymphocyte ratio mirror. J Biomark 2016; 2016: 8160393.

6. Kim JM, Moon J, Ahn SW. The etiologies of early neurological deterioration after thrombolysis and risk factors of ischemia progression. J Stroke Cerebrovasc Dis 2016; 25: 383-388.

7. Dawood FZ, Judd S, Howard VJ. High-sensitivity creactive protein and risk of stroke in atrial fibrillation (from the reasons for geographic and racial differences in stroke study). Am J Cardiol Epub 2016.

8. Li J, Zhao X, Meng X. High-sensitive c-reactive protein predicts recurrent stroke and poor functional outcome: subanalysis of the clopidogrel in high-risk patients with acute nondisabling cerebrovascular events trial. Stroke 2016; 47: 2025-2030.

9. Rocco A, Ringleb PA, Grittner U. Follow-up C-reactive protein level is more strongly associated with outcome in stroke patients than admission levels. Neurol Sci 2015; 36: 2235-2241.

10. Elkind MS, Luna JM, McClure LA. LIMITS Investigators. C-reactive protein as a prognostic marker after lacunar stroke: levels of inflammatory markers in the treatment of stroke study. Stroke 2014; 45: 707-716.

11. Seo WK, Seok HY, Kim JH. C-reactive protein is a predictor of early neurologic deterioration in acute ischemic stroke. J Stroke Cerebrovasc Dis 2012; 21: 181-186.

12. Ryu WS, Schellingerhout D, Jeong SW. Association between serum lipid profiles and early neurological deterioration in acute ischemic stroke. J Stroke Cerebrovasc Dis 2016; 25: 2024-2030.

13. Soliman EZ, Howard G, Meschia JF. Self-reported atrial fibrillation and risk of stroke in the Reasons for Geographic and Racial Differences in Stroke (REGARDS) study. Stroke 2011; 42: 2950-2953.

14. Ntaios G, Lip GY, Lambrou D. Leukoaraiosis and stroke recurrence risk in patients with and without atrial fibrillation. Neurology 2015; 84: 1213-1219.

15. Pearson TA, Mensah GA, Alexander RW. Centers for Disease Control and Prevention; American Heart Association. Markers of inflammation and cardiovascular disease: application to clinical and public health practice: a statement for healthcare professionals from the Centers for Disease Control and Prevention and the American Heart Association. Circulation 2003; 107: 499-511.

16. The Warfarin-Aspirin Symptomatic Intracranial Disease (WASID) Study Group. Prognosis of patients with symptomatic vertebral or basilar artery stenosis. Stroke 1998; 29: 1389-1392.

17. North American Symptomatic Carotid Endarterectomy Trial (NASCET) Steering Committee. North American symptomatic carotid endarterectomy trial. Methods, patient characteristics, and progress. Stroke 1991; 22: 711-720.

18. Liu X, Xu G, Wu W, Zhang R, Yin Q, Zhu W. Subtypes and one-year survival of first-ever stroke in Chinese patients: The Nanjing Stroke Registry. Cerebrovasc Dis 2006; 22: 130-136.

19. Adams HP, Del ZG, Alberts MJ. Guidelines for the early management of adults with ischemic stroke: a guideline from the American Heart Association/American Stroke Association Stroke Council, Clinical Cardiology Council, Cardiovascular Radiology and Intervention Council, and the Atherosclerotic Peripheral Vascular Disease and Quality of Care Outcomes in Research Interdisciplinary Working Groups: the American Academy of Neurology affirms the value of this guideline as an educational tool for neurologists. Stroke 2007; 38: 1655-711.

20. Duan Z, Fu C, Chen B. Lesion patterns of single small subcortical infarct and its association with early 
neurological deterioration. Neurol Sci 2015; 36: 1851-1857.

21. Zuowei Duan, Wen Sun, Wenhua Liu. Acute DWI lesion patterns predict progressive small subcortical infarct in the perforator territory of middle cerebral artery. Int J Stroke 2015; 10: 207-212.

22. Simonsen CZ, Schmitz ML, Madsen MH. Early neurological deterioration after thrombolysis: Clinical and imaging predictors. Int J Stroke 2016; 11: 776-782.

23. Kim YD, Choi HY, Jung YH, Yoo J. The ischemic stroke predictive risk score predicts early neurological deterioration. J Stroke Cerebrovasc Dis 2016; 25: 819-824.

24. Rost NS, Wolf PA, Kase CS. Plasma concentration of Creactive protein and risk of ischemic stroke and transient ischemic attack: the Framingham study. Stroke 2001; 32: 2575-2579.

25. Lip GY, Patel JV, Hughes E. High-sensitivity C-reactive protein and soluble CD40 ligand as indices of inflammation and platelet activation in 880 patients with nonvalvular atrial fibrillation: relationship to stroke risk factors, stroke risk stratification schema, and prognosis. Stroke 2007; 38: 1229-1237.

26. Nilupul Perera M, Ma HK, Arakawa S, Howells DW, Markus R, Rowe CC, Donnan GA. Inflammation following stroke. J Clin Neurosci 2006; 13: 1-8.
27. Licata G, Tuttolomondo A, Di Raimondo D. Immunoinflammatory activation in acute cardio-embolic strokes in comparison with other subtypes of ischemic stroke. Thromb Haemost 2009; 101: 929-937.

28. Azar RR, Kassab R, Zoghbi A. Effects of clopidogrel on soluble CD40 ligand and on high-sensitivity C-reactive protein in patients with stable coronary artery disease. Am Heart J 2006; 151: 521.

29. Weber M, Bhatt DL, Brennan DM. CHARISMA investigators. High-sensitivity C-reactive protein and clopidogrel treatment in patients at high risk of cardiovascular events: a substudy from the CHARISMA trial. Heart 2011; 97: 626-631.

\section{*Correspondence to}

Xinjiang Zhang

Department of Neurology

The First People's Hospital of Yangzhou

PR China 